\title{
Eyebrow transplant
}

\author{
Viroj Vong, MD iD \\ H.H.H. Hair Transplant Center, Bangkok, Thailand
}

Eyebrow compose of very unique characteristics hair. Only hair from lower nape of occipital scalp or very light pubic hair can match loosely as donor hair. Blonde hair or light color hair give better result. Black Asian hair is more difficult because of the contrast between skin and hair. Design eyebrow follow natural pattern, gender, direction within normal variation is very important to make it look natural. Donor hair can be done by 2 methods. Common one is H.H.H. FUE. FUE is done by extract each unit of hair follicle out from occipital scalp then transplant to eyebrow. Second is Strip harvesting by remove a piece of occipital scalp divide to follicular unit. Then transplant this unit to eyebrow. FUE produce no scar at the occipital scalp (Donor site). Strip harvesting produce a linear scar at the donor site (occipital scalp).

Keywords: eyebrow; hair transplant

\section{Introduction}

The patient was white, male, and 75 years old. He was not happy with his eyebrows and thought they were too thin. He recovered from Crohn's disease of his colon. At the time of the visit, his disease was inactive since $>5$ years and was not treated with medications. Crohn's disease affected not only his colon but also his eyebrows, mustache, and beard, where he lost significant amounts of hair. Thus, he wanted to restore his eyebrows to its state when he was young. He designed his eyebrows the way he liked, including the length from the nose to the tail of the eyebrow. The tail end was a little beyond the lateral canthus and directed upward. He wanted to have the same proportion as that of the eyebrows of men and the upswing tail and overall shape to appear like a Chinese sword. He also wanted to increase the density of the whole eyebrow. He wanted this design because it matches with his triangular shape frontal hairline and the original shape of his eyebrows. After some minor adjustment, the surgery began after a general physical examina- tion and blood test. He consulted his gastrointestinal specialist in Britain, who confirmed that he was safe to undergo hair transplantation. His vital signs and blood test results for human immunodeficiency virus (HIV), bleeding time, and coagulation time were all within their normal limits. He signed the consent form for surgery.

\section{Case report}

\section{Shampoo before surgery}

His face was cleansed, and his scalp was washed with shampoo with strong antiseptics, thoroughly. His hair was dried before returning to the design chair.

\section{Design confirmation}

He drew the boundary and position of his new eyebrows again. After indicating satisfaction of the eyebrow design, he was brought to the operating room (Fig. 1A).

Received November 7, 2019; Revised December 27, 2019; Accepted December 28, 2019

Corresponding author: Viroj Vong

E-mail: hhh.hair@gmail.com

This is an Open Access article distributed under the terms of the Creative Commons Attribution Non-Commercial License (http://creativecommons.org/licenses/by-nc/4.0), which permits unrestricted non-commercial use, distribution, and reproduction in any medium, provided the original work is properly cited.

Copyright (C) 2020 Korean Society of Korean Cosmetic Surgery and Medicine (KSKCS \& KCCS). 


\section{Anesthesia}

Surgery was performed under local anesthesia induced with $0.5 \%$ xylocaine mixed half and half with $0.125 \%$ Marcaine for both donor sites (occipital scalp and lower nape area) and eyebrows. The total solution was approximately $30 \mathrm{ml}, 10 \mathrm{ml}$ for the eyebrow and $20 \mathrm{ml}$ for the occipital scalp. The operation was performed under aseptic conditions. The surgical site was cleansed with strong antiseptics such as iodine and alcohol.

\section{Skin crease slit of the recipient site (eyebrows)}

The skin crease slit was performed following the skin crease and against the direction of the hair (Fig. 1B, C). A slit was precut with a $0.7-\mathrm{mm}$ width micro-blade. The blade was bent approximately $45^{\circ}$ and inserted with a close angle against the natural exit angle. To ensure that the follicular unit (FU) remains in the site with a correct hair curl, the blade was held up or down following the natural curl of the eyebrow. The number of slits was 309 in the right eyebrow and 315 in the left eyebrow [1-5].

\section{Donor harvesting}

After all the slits were made, H.H.H. FUE was started. Each follicle was manually extracted using a 0.8 - to $1.0-\mathrm{mm}$ punch, that is, $0.8 \mathrm{~mm}$ for each hair follicle and $1.0 \mathrm{~mm}$ for 2 or 3 hair follicles. Deep insertion and rotation were performed during extraction to include perifollicular fibro-adipose tissue. The extracted follicles were put in disc containing Ringer's lactate and platelet-rich plasma (PRP) solutions. The follicles were examined under microscopy and divided 3-haired to 1- or 2-haired follicles.

\section{Implantation}

One-haired follicles were implanted for the nasal edge and tail of the eyebrows, and 2-haired follicles were implanted for the mid body. FUs were inserted in the pre-made slits manually
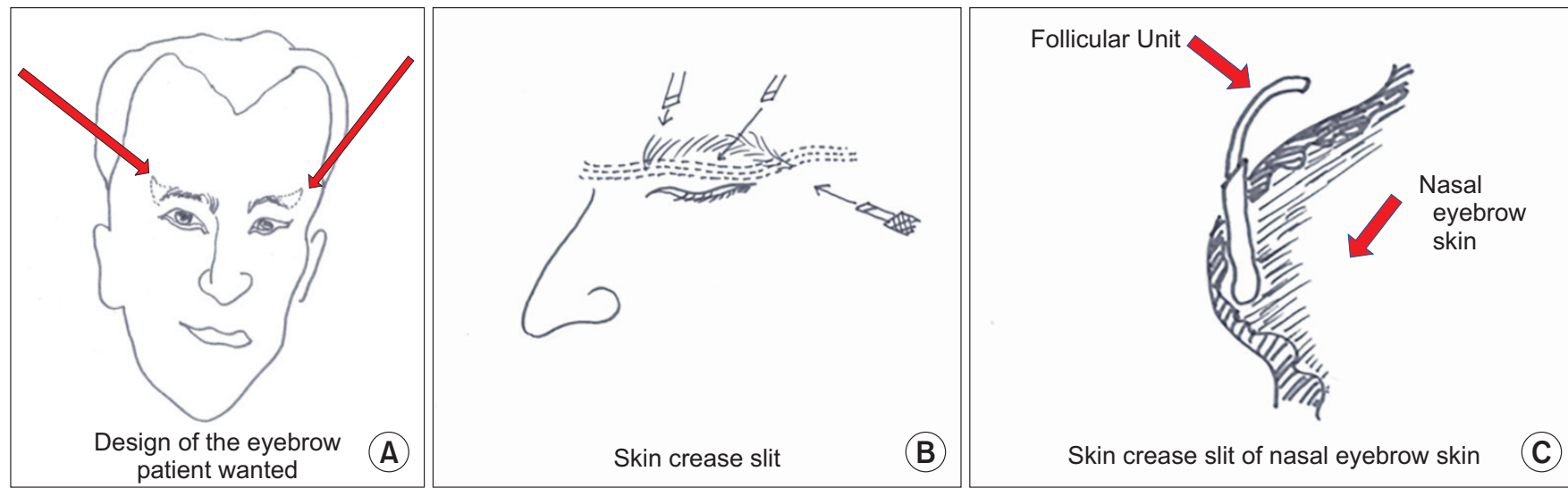

Fig. 1. (A-C) Eyebrow transplant procedure.
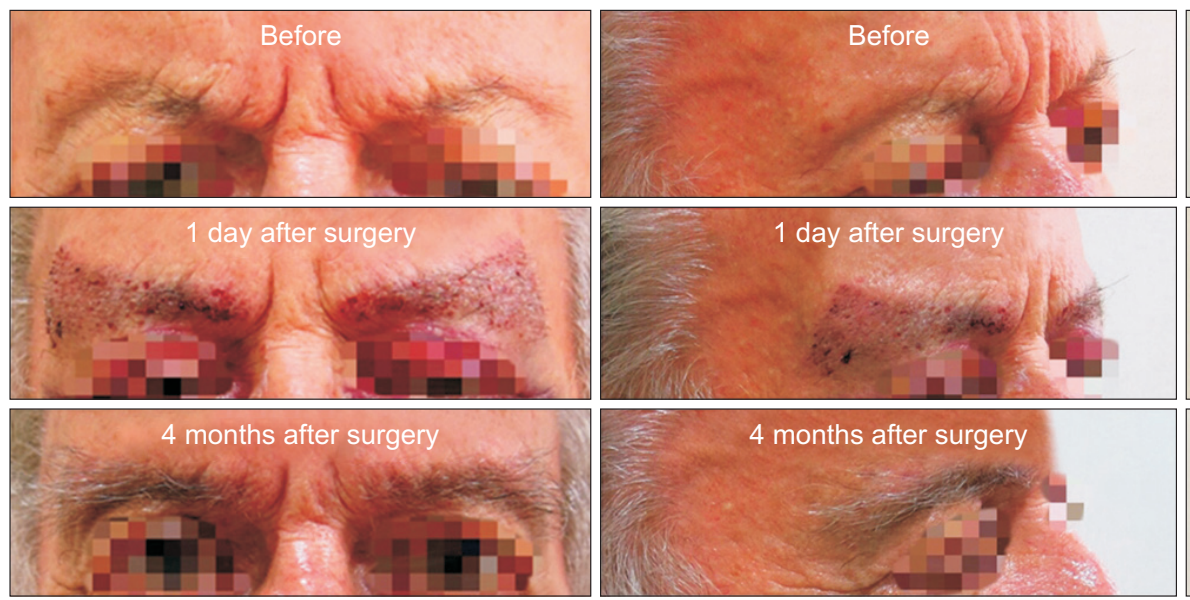

Fig. 2. Before, 1 day after, and 4 months after transplantation. 
with micro forceps. The whole process was performed gently and fast enough to minimize crushing of tissue. The FUs were grabbed and inserted with the correct hair curl one by one. The shape and position of the eyebrows, and level and evenness of the insertion were rechecked thoroughly.

\section{Postoperative care}

To dress the fresh wound, bacitracin ointment was applied on both eyebrows and the donor wound. For the eyebrows, no gauze dressing was applied. However, gauze dressing was applied to the donor wound to prevent blood and serum oozing. He returned home with pain medications such as tramadol and antibiotics (dicloxacillin), and wore large sunglasses to protect his eyebrows for 2 weeks. He slept upright for 2 weeks to prevent any damage to the new eyebrows. The next morning, he returned to the clinic for cleansing, shampooing, rechecking, and dressing of the new eyebrows. He had to follow this regimen for 14 more days before he could return to his normal activities.

\section{Follow-up}

After around 2 weeks, some new hair started falling, which is called surgically induced catagen (acceleration of the catagen phase of the hair cycle) or falling hair called telogen hair. After 3 months, new hair started to grow. Four months later (most commonly 8 to 12 months to attain good esthetics), he returned to the clinic and showed his new eyebrows, of which he was highly satisfied. More hair grew until 8 to 12 months after transplantation (Fig. 2).

\section{Discussion}

The 3 parts of the eyebrow have different characteristics in terms of origin and shape (Fig. 3). The eyebrows originate from the nose, forehead, and eyes. The nasal portion of the eyebrow is from the nose, with upright hair because of pulling from the corrugator muscle. The frontal portion is from the forehead, with oblique hair because of the pulling from the frontal muscle. The eye portion is from around the eyes. Hair is pulled by the muscle around the eyes.

The variation and shape of the eyebrows significantly affect the vellus hair pattern on the forehead (Fig. 4).

\section{Etiology of eyebrow los [6]}

\section{Donor Harvesting for H.H.H. FUE}

Donor hair can be harvested using 2 methods, strip and FUE. Currently, H.H.H. FUE is the most common and effective method for producing less transection, increasing growth, preventing scarring at the donor site. Although the strip harvesting method produces a linear scar on the occipital scalp, it is still used. The eyebrows can be accentuated or embellished. However, the eyebrows require more maintenance after than before transplantation because the transplanted hair continue to grow.

\section{Extracted FU (H.H.H. FUE) from the donor site}

The donor site can be the occipital scalp, beard, chest, stomach, belly button, pubic area, and so on. The FU was extracted and then transplanted to the eyebrow, bald site, or scalp. If the
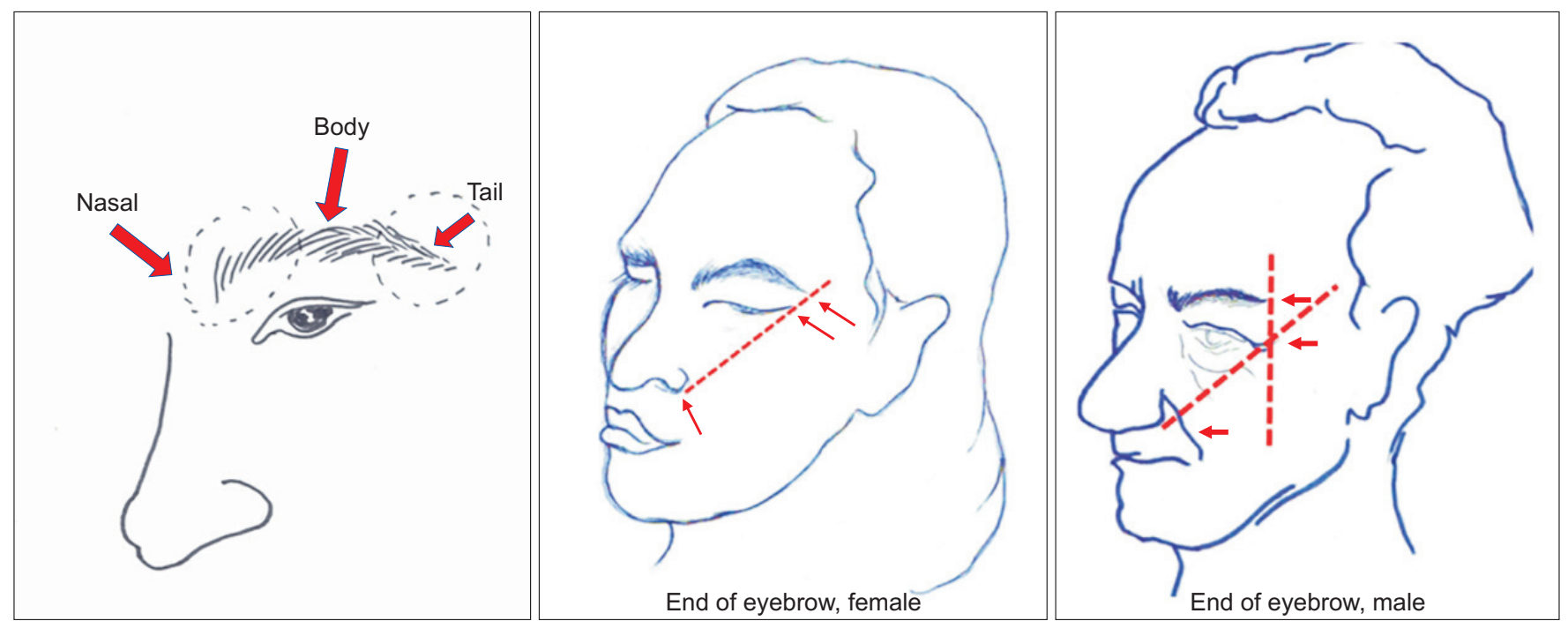

Fig. 3. Anatomy of eyebrow. 


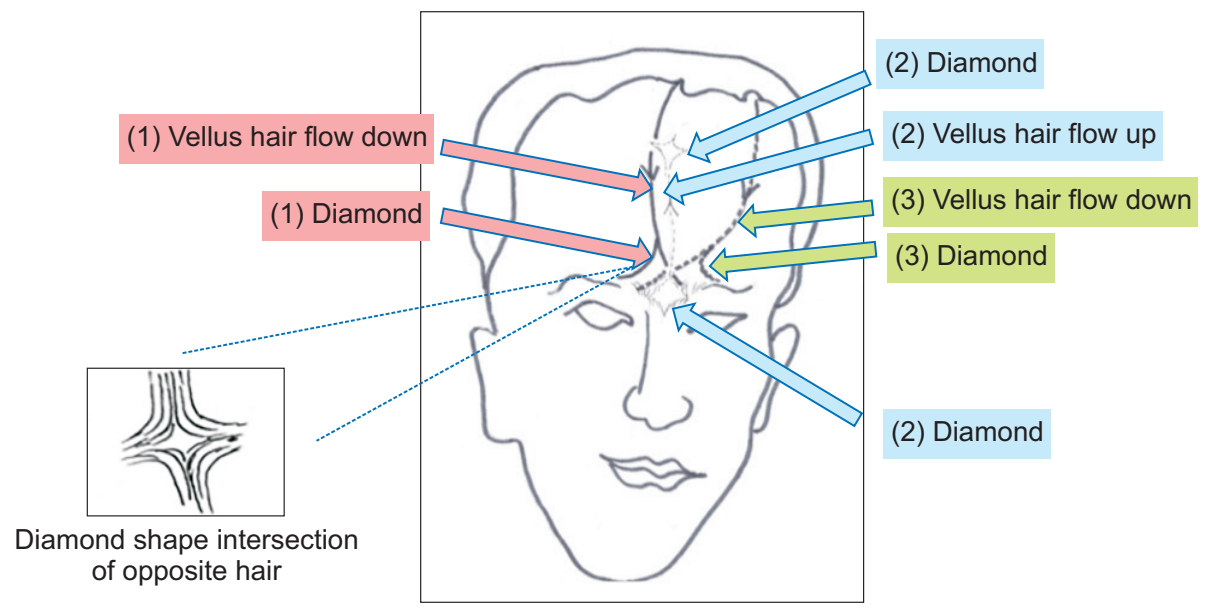

Fig. 4. Patterns of vellus hair flow down and diamond shape in nasal portion. (1) Midline with no bifurcation (red colored arrows). If one have a midline natural partline (born with). The part line extend downward to the nose. Vellus hair grow along this line. Flow down along the frontal portion of the eyebrow and divert above nasal portion of the eyebrow produce diamond shape intersection of opposite hair at above the base of the nose. No bifurcation of nasal portion. (2) Midline bilateral bifurcation (blue colored arrows). Nasal hair from both eyebrow flow up and converge at midline produce the bifurcation and diamond shape and continue midline then start divert again produce another diamond shape at upper frontal forehead. Vellus hair and terminal hair grow along this line. Only the terminal dark hair that can be seen easily. Medial portion of the nasal hair flow down to the mid nose produce the tip of the lower diamond. (3) Lateral pattern (green colored arrows) (left or right, left are more common). One who have a partline on left side of forehead. The partline will extend downward and medial ward. This line divert out and join at nasal portion of both eye produce a very asymmetrical diamond shape. Left nasal portion produce a bifurcation. Right nasal portion produce normal shape.

FU is transplanted to the face, then new beard, mustache, goatee, and side burn can be reconstructed.

\section{H.H.H. FUE is different from other methods for the following reasons}

H1. The gentle and sharp hand punch minimizes trauma during follicle tissue cutting. No gigantic robot is required. No motorized electric machine is used for fast cutting, preventing shearing damage to the tissue. Trained hands are more precise in extraction and insertion than a robot, which causes transection, crushing, drying, and injury, among others. The hand can go deep to include fibroadipose perifollicular tissue and minimum trimming during examination under microscopy.

H2. Robots do not have emotions, so they are not cable of soft and gentle handling of tissues, unlike humans.

H3. The holding solution to energize the hair follicles contains Ringer's lactate solution with PRP.

After FUE, the surgical wound leaves no scar on the scalp.

\section{Why the skin crease slit?}

Sagittal and coronal slits are artificial. The natural collagen line is never scientifically parallel or perpendicular. If used, these slits cause more trauma to the collagen bundle and produce more scarring and blood loss because the skin crease is 1 , a minimal tension line; 2 , is a natural line; 3 , produces minimal blood loss during slit creation; and 4, produces minimal scarring after operation. The normal collagen bundle is arranged almost parallel to the skin crease in the dermis. If slits are made through and parallel to the bottom of the crease, these will separate the bundle with a natural cleavage plane. During implantation, the FU stays in the naturel plane or the pocket created. Attachment of an erector pilli muscle to the skin crease creates a natural appearance. Thus, scarring is minimized and a natural look is attained after hair contraction, both during facial expression and hair erection. The pulling by the muscle decreases the exit angle, producing a close angle between the new eyebrow and skin.

\section{Considerations in this case}

As a natural eyebrow has unique characteristic hair, a matching donor hair for eyebrow transplantation is difficult to find. Generally, the sources of donor hair are 1, those at the lower nape at the end of the scalp, which has thin and soft but too straight hair; and 2, the pubic hair, which is thicker than the normal eyebrow, had good curvature but slow growth. The donor hair is then transplanted to the eyebrow region in a similar manner as that in transplanting occipital hair.

The eyebrow is the most sensitive hair on the face, so transplantation must be meticulously and carefully performed. Attention should be paid to the direction and curl of the hair. The 
thinnest hair strand is selected for the nasal portion, edge, and tail of the eyebrow. The exit angle at the nasal portion has to be flat. Bending the cutting blade will help achieve a flat angle. The curl is kept as natural as possible. The length of the new hair is important. The normal eyebrow is short, but the donor hair is from the occipital scalp. Hair in the new eyebrow continue to grow very long, so the new eyebrow needs to be trimmed, accentuated, or embellished often. In a personal discussion, Dr. Arambulo, a hair transplant specialist and dermatologist from the Philippines who have performed many eyebrow transplants, reported that the transplanted hair requires frequent trimming only in the beginning 1 to 2 years after transplantation because the recipient site influences hair growth. The new hair slowly adjusts to its new location. Occipital hair becomes true eyebrows. In women, female hormones influence the eyebrow by making the transplanted hair softer and better.

\section{Conflicts of interest}

The author has nothing to disclose.

\section{References}

1. Vong V. Normal hairline. Hair Transplant Forum Int 1999;9: 178.

2. Vong V. Whorl on scalp of Thai monk and nun. Hair Transpl Forum Int 2005;15:7.

3. Vong V. Line Thai micro hairline. Paper presented at: ISHRS 15th Annual Meeting; 2007 Sep 30; Las Vegas, NV.

4. Vong V. Line Thai micro hairline. Hair Transpl Forum Int 2008;18:48.

5. Vong V. Beard hair transplant to the hairline. J Cosmet Med 2017;1:63-7.

6. James WD, Berger TG, Elston DM. Andrews' diseases of the skin: clinical dermatology. 10th ed. Philadelphia, PA: Saunders Elsevier; 2006. p. 579 